\title{
The Eddington Mission
}

\author{
Ian Roxburgh \\ Astronomy Unit, Queen Mary, University of London, E1 4NS, UK \\ Observatoire de Paris, Place Jules Janssen, 92195 Meudon, France
}

\author{
Fabio Favata \\ Astrophysics Division, ESTEC, ESA, Noordwijk, The Netherlands
}

\begin{abstract}
The Eddington mission was given full approval by the European Space Agency on the 23rd May 2002, as part of the new "Cosmic Vision" Science programme, with launch scheduled for 2007. Its twin science objectives are asteroseismology and planet finding. In its current design it consists of $4 \times 60$ $\mathrm{cm}$ folded Schmidt telescopes, each with $5^{\circ} \times 5^{\circ}$ field of view and its own CCD array camera. The current observing plan is to spend 2 years primarily devoted to asteroseismology with 1-3 months on different target fields monitoring up to 50,000 stars per field, and 3 years continuously on a single target field monitoring upwards of 100,000 stars as required for planet searching. The asteroseismic goal is to be able to detect oscillations frequencies with a precision $0.1-0.3 \mu \mathrm{Hz}$.
\end{abstract}

\section{The Eddington Mission}

\subsection{Overview}

The Eddington mission was proposed in early 2000 to ESA in response to the "Call for mission proposals for two flexi-missions (F2 and F3)", released in October 1999 in the context of the Horizons 2000 programme. The proposal was submitted by an international scientific team led by Roxburgh, ChristensenDalsgaard and Favata (2000). It built on the work done over two decades on previous proposals that had been studied within ESA and CNES ( EVRIS, PRISMA, PRISMA2, STARS). The mission has two complementary primary scientific aims, to produce the data on stellar oscillations necessary for understanding the interior structure, dynamics and evolution of stars, and to detect and characterise habitable planets around other stars.

Both scientific goals will be achieved by performing highly accurate timeresolved photometry on a large number of stars, with a simple payload which is essentially a wide field broad-band photometer. The accurate light curves will be used to determine stellar oscillation frequencies, and thus allow the use of asteroseismic tools to probe the interior structure of stars, quantitatively determining, e.g., the size of convective regions, the structure of regions with steep changes in chemical composition and internal rotation, and to therefore determine very accurate stellar ages. At the same time, planets as small as the Earth orbiting the target stars in the habitable zone will be found through the temporary drops in the stellar light caused by their transits. 
Following the favourable reviews by ESA's scientific advisory bodies (the Astronomy working Group \& the space science Advisory Committee) Eddington was selected in March 2000 as one of the 4 missions for which an assessment study carried out in the course of the spring and summer of 2000 (Favata et al. 2000). Following presentation of the studies in September 2000, Eddington was selected with a "reserve status". Study activities continued in the course of 2001 and 2002, and in May 2002 Eddington was approved by ESA's Science Programme Committee to be implemented in the framework of the $H$ erschel and Planck projects in ESA's rescoped science programme Cosmic Vision.

\subsection{Data Rights and Observing Programme.}

Eddington is a facility-type mission, for which the observing plan will be the result of a combination of an open, competitive proposal cycle with a broad community consulting process. Astroseismic targets should include a wide variety of stars with different characteristics and in different stages of evolution; these could include open clusters with massive pulsating stars, open clusters with solar-like pulsators, old metal poor stars, and the 3 year planet search field. $E$ ddington is not a PI led mission and the data will be made available to the wider scientific community. Groups and consortia may propose (in response to an Announcement of Opportunity) to be approved to have access to all or part of the data, and we anticipate that several groups will be given such approval. Approved groups will then have priority rights for 1 year, following which the data will be made public.

\subsection{Payload Configuration and Data Products}

The primary $E$ ddington data product is a set of relatively calibrated photometric light curves for each star in the field of view down to a predefined magnitude limit. To maximize the field of view and collecting area the payload is baselined to consist of 4 parallel very similar (possibly identical) instruments, each of them independently producing a light curve, which will be merged a posteriori. Each of the 4 instruments consists of 3 basic components: (1) a wide field optical telescope, (2) a mosaic CCD camera (named "EddiCam") and (3) a data processing unit (DPU).

\subsection{Mission Procurement}

ESA will have overall responsibility for the mission, and will in particular be responsible for the procurement of the spacecraft and of the ground segment, for the integration of the payload and spacecraft units, for the launch and operations, the acquisition of the data, their reduction, archiving and their distribution to the holders of the data rights. The payload is planned to be procured through a partnership between ESA and a consortium of scientific institutes.

ESA is planned to operate the Scientific Operations Centre (SOC) which will process and archive the data from the instrument and deliver the scientific data products (relatively calibrated light curves) to the holders of the data rights. 


\section{The Current Mission Concept}

The final form of the mission will be decided following detailed industrial studies and advice from the $E$ ddington Science Team. The current working concept is as follows:

$4 \times 0.6 \mathrm{~m}$ folded Schmidt telescopes each with $5^{o} \times 5^{o}$ field of view.

Mosaic CCD "panoramic" cameras with $6 \mathrm{E} 2 \mathrm{~V}$ 42-C0 chips.

Orbit at L $2-95 \%$ duty cycle -5 year mission.

3 years on 1 field (planet finding + asteroseismology).

$1-3$ months on asteroseismology fields (total 2 years).

Launch: 2007.

\section{Asteroseismology Fields}

Max. no. of stars per field: $2000\left(m_{v}<11\right), 50,000\left(m_{v}<15\right)$.

Precision on frequencies: $0.1 \mu \mathrm{Hz}$ (goal), $0.3 \mu \mathrm{Hz}$ (bottom line).

Frequency range: $1 \mu \mathrm{Hz}-100 \mathrm{mHz}$ (goal), $1 \mu \mathrm{Hz}-10 \mathrm{mHz}$ (bottom line).

Magnitude range: 3-15 (goal), 5-15 (bottom line).

Time sampling: $0.5 \mathrm{sec}-30 \mathrm{sec}$

Chromatic information: to be determined.

Planet Finding Field (also available for asteroseismology)

No. of stars: $>100,000\left(m_{v}<17\right)$.

Time sampling: $30 \mathrm{sec}-$ less for bright stars

\section{Acknowledgements}

We wish to record our thanks to the many scientists who have contributed over two decades to convincing ESA to fly an asteroseismology mission by working on the precursor proposals PRISMA, PRISMA2 and $S$ TARS, and especially to those who worked for the selection of $E$ ddington. In particular we record the contribution to Eddington made by the Assessment Study Team, the successor Eddington Science Team, and technical support from ESA.

\section{References}

Favata F., Roxburgh I. W. and Christensen-Dalsgaard J. eds, 2000. with Aerts C., Antonello E., Catala C., Deeg H., Gimenez A., Grenon M., Pace O., Penny A., Schneider J., Waltham N., Eddington, A Mission to Map Stellar Evolution through Oscillations and to Find Habitable Planets, Report of Assessment Study, ESA-SCI(2000)8

Roxburgh I. W., Christensen Dalsgaard J. and Favata F., eds 2000. with Antonello E., Baade D., Badiali M., Baglin A., Bedding T., Brown T., Catala C., Collier A., Dziembowski W., Gilmore G., Gimenez A,, Gough D., Horne K., Kjelsden H., Leger A., Penny A., Preite-Matinez A., Rivinus Th., Schneider J., Stefl Z., Sterken C., Weiss W. Eddington - A Stellar Physics and Planet Finder Explorer. Proposal submitted to ESA in response to the ESA F2/F3 call for proposals. 